\title{
Horizons/Théâtre
}

Revue d'études théâtrales

12 | 2018

Les dramaturgies arabes et l'Occident

\section{L'essor du théâtre occidental dans l'Égypte khédiviale et les premières aspirations des Égyptiens à un théâtre moderne arabe}

Ons Trabelsi

\section{(2) OpenEdition \\ 1 Journals}

Édition électronique

URL : http://journals.openedition.org/ht/292

DOI : $10.4000 /$ ht.292

ISSN : 2678-5420

Éditeur

Presses universitaires de Bordeaux

\section{Édition imprimée}

Date de publication : 1 janvier 2018

Pagination : 26-46

ISSN : 2261-4591

\section{Référence électronique}

Ons Trabelsi, «L'essor du théâtre occidental dans l'Égypte khédiviale et les premières aspirations des Égyptiens à un théâtre moderne arabe », Horizons/Théâtre [En ligne], 12 | 2018, mis en ligne le 01 janvier 2019, consulté le 20 juillet 2019. URL : http://journals.openedition.org/ht/292 ; DOI : 10.4000/ ht.292

\section{(c) (i) $\odot$}

La revue Horizons/Théâtre est mise à disposition selon les termes de la Licence Creative Commons Attribution - Pas d'Utilisation Commerciale - Pas de Modification 4.0 International. 


\section{ONS TRABELSI}

Ons Trabelsi est docteure en arts du spectacle, elle a soutenu sa thèse intitulée "Sídĩ Molière. Traductions et adaptations de Molière en arabe: Liban, Égypte, Tunisie (1847-1967) » sous la direction de Christian Biet, en juin 2017 à l'Université Paris Nanterre-école doctorale "Lettres, langues et spectacles ». Elle est actuellement attachée temporaire d'enseignement et de recherche au département de Langues et Cultures étrangères, à l'Université de Lorraine.

Mail: ons.trabel@gmail.com

Résumé: L'histoire de la naissance et de l'essor du théâtre arabe moderne est indissociable du contexte politique et culturel de la naissance de l'Égypte moderne. Elle est également liée à la figure du Khédive Ismail. La construction des théâtres, l'invitation des troupes étrangères ainsi que la presse de langue arabe permettent alors d'initier le

Abstract: The story of the birth and rise of modern Arab theater is inseparable from the political and cultural context of the birth of modern Egypt. She is also associated with the figure of Khedive Ismail. The construction of the theaters, the invitation of the foreign troops as well as the Arabic language press made it possible to initiate the Arab public public arabe à la dramaturgie occidentale et de créer la volonté et l'ambition d'avoir un théâtre propre aux Égyptiens.

Mots-cLÉs: Théâtre arabe, dramaturgie occidentale, pouvoir, comédie française, initiation, appropriation, public

to the Western dramaturgy and to create the will and the ambition to have a theater proper to the Egyptians.

Keywords: Arab theater, Western dramaturgy, power, French comedy, initiation, appropriation, public 


\section{L'essor du théâtre occidental dans l'Égypte khédiviale et les premières aspirations des Égyptiens à un théâtre moderne arabe}

Nous PROposons D'ÉTUdier la naissance et le développement du théâtre moderne en Égypte. L'histoire politique ne sera évoquée qu'en tant que toile de fond, indispensable, pour la compréhension des relations culturelles entre la France et l'Égypte ainsi que pour la compréhension des différentes initiatives de l'élite égyptienne et son rapport au pouvoir. Nous étudierons par la suite, l'importance du rôle joué par les journalistes et les premiers dramaturges égyptiens dans l'initiation du public à la dramaturgie occidentale. Cette initiation sera donc présentée à travers les initiatives personnelles comme le Projet d'un théâtre national de Mohammed Onsy et de Louis Farugia, ainsi que les annonces des journaux égyptiens, comme le journal Wādì al-Nìl qui encourage les initiatives de traductions (Rifaa't at-Tahtawi, 'Uthmān Jalāl, al- Muaylīhī), traduit les affiches des spectacles italiens pour le public local, explique les nouveaux termes dramatiques (Opéra, Comédie) et tente de rendre moins étrange les spectacles et de trouver des références communes aux publics arabe et occidental.

\section{L'essor du théâtre sous l'Égypte khédiviale}

Ismail Pacha ${ }^{1}$ règne de 1863 à 1879 sur un pays en pleine effervescence et une société en pleine mutation. Les avis sur la période de son règne sont controversés. Il est présenté, à la fois, comme le bâtisseur de l'Égypte moderne et comme le premier responsable de la ruine du pays. En effet, le khédive avait transformé l'Égypte par des réalisations économiques, sociales et culturelles remarquables, mais il a également entrainé son pays dans une crise financière et politique qui a ouvert la porte à la domination coloniale. Cette dynamique de transformations urbaine, culturelle et sociale, les échanges entre la France et l'Égypte à cette époque et les différentes interactions culturelles et artistiques qui en résultent, constituent le cadre dans lequel se développera le théâtre arabe de répertoire français. Le théâtre moderne naissant représente alors largement ces mutations sociales et se trouve directement concerné par le pouvoir du Khédive Ismail. Un pouvoir qui oscille entre l'en- 
couragement, l'accueil, les subventions, l'exil, la construction et la fermeture des théâtres. Les auteurs, traducteurs, adaptateurs seront eux aussi partagés entre éloge et critique à travers leurs pièces ou leurs écrits journalistiques. Ainsi, Ismail ne représente pas pour cette époque un simple Vice-roi mais un personnage politique imposant qui fera l'objet de représentations satiriques et théâtrales.

Le Khédive se donne comme mission d'occidentaliser son pays en déclarant: «Mon pays n'est plus en Afrique. Nous faisons partie de l'Europe. » Cette phrase célèbre a été longtemps citée même après sa mort. Dans les années 1910, elle figurera chaque jour en première page du Journal du Caire, l'un des principaux quotidiens francophones de la capitale. Nous ne pouvons pas savoir si le khédive l'a réellement prononcée, mais « elle correspond parfaitement à son état d'esprit ${ }^{2} \gg$. En tout cas cette phrase exprime la volonté du souverain de réorganiser son pays, d'accélérer sa modernisation dans les domaines culturels, commerciaux et militaires et de s'ouvrir davantage sur l'Europe et en particulier sur la France. Il lance alors plusieurs projets. En peu d'années, l'Égypte connaît une croissance sans précédent ${ }^{3}$. Le Caire médiéval devient une ville moderne capable d'accueillir les invités étrangers lors de l'inauguration du Canal de Suez et de donner une image du prestige et du rayonnement de son règne. La ville se transforme et s'embellit grâce à l'aménagement de plusieurs places et la création de nouveaux quartiers et devient un Paris en miniature, une ville de boulevards et de ballets, de casinos et de cafés chantants.

Le Khédive rénove l'École des langues instituée par Méhémet Ali, qui reprend alors ses activités et forme les meilleurs journalistes et traducteurs de l'époque dont 'Uthmān Jalāl qui traduit en arabe les œuvres de La Fontaine, Bernardin de Saint-Pierre, Racine, Corneille, et Molière, ainsi qu'Abū-l-su' ūd, fondateur du journal $W a \bar{d} \bar{\imath}$ al-Nìl$l^{4}$. Ce journal officiel ${ }^{5}$ a largement contribué à l'essor des lettres. Distribué gratuitement aux élèves, il publiait des articles à caractère littéraire, social, et scientifique rédigés par des professeurs renommés. L'expansion de l'enseignement, même si elle reste limitée aux grandes villes notamment Le Caire et Alexandrie, prépare une nouvelle génération d'Égyptiens appartenant à une classe moyenne constituée de professeurs, de journalistes, de médecins et de fonctionnaires. Elle permet à certains Égyptiens d'accéder à la classe dirigeante et à des postes élevés qui étaient naguère l'apanage des seuls Turcs et étrangers. L'éducation des filles contribue également à cette mutation sociale et donne à la femme égyptienne un 
nouveau statut et une place dans la nouvelle configuration de la ville et de la société.

Le khédive appelle un grand nombre d'étrangers pour venir s'installer dans le pays. Les étrangers vivant en Égypte adoptent un nouveau type de vie mondaine fait de sorties, de café-concert, de jeux et de restaurants. Le contact direct avec des étrangers provoque au début l'étonnement des Égyptiens avant de se muer en imitation. Avec les transformations économiques et culturelles qu'a connues le pays, une nouvelle classe sociale émerge et adopte ce mode de vie à l'occidentale. L'attachement du Khédive lui-même à la France fait de la vie parisienne le modèle à suivre et accentue l'occidentalisation des mœurs.

Le contexte politique de la naissance de l'Égypte moderne, les réformes, la construction des écoles et la naissance de la presse sont des facteurs directs qui ont favorisé la diffusion de la littérature dramatique occidentale. Le théâtre occidental, ne sera plus, sous le règne du Khédive Ismail, un simple divertissement étranger. En effet, son règne sera particulièrement marqué par les constructions des salles de théâtre, les invitations des troupes étrangères, et surtout par la mise en place d'une nouvelle tradition théâtrale. Le nouveau caractère cosmopolite des villes égyptiennes et la diffusion de l'enseignement sont des facteurs qui ont joué un rôle important dans la naissance d'une classe moyenne et d'un public qui peut se permettre d'aller au théâtre et de découvrir le répertoire occidental.

Au Caire, l'activité culturelle était concentrée dans la partie d'al-Ezbékieh ${ }^{6}$. L'endroit était abandonné jusqu'au retour d'Ismail Pacha de Paris en 1867. Au début, Ismail y a fait construire des promenades et des jardins où il est permis aux passants de se promener le soir seulement en écoutant des groupes de musique occidentale jouer toute la nuit. Après la construction des théâtres et à partir de 1871, il fut désormais permis de s'y promener de jour comme de nuit et ce fut l'endroit principal des cérémonies officielles des communautés étrangères comme la commémoration du Quatorze Juillet. Les jardins étaient très fréquentés grâce aux activités théâtrales et musicales de ces communautés 7 . Le Théâtre de la Comédie Française est le premier théâtre construit dans le jardin situé au sud de la place Ezbékieh. Il est inauguré le 4 janvier 1869 par La Belle Hélène d'Offenbach, avec la présence du Khédive et son fils Tawfīq. Le théâtre était construit en bois et richement embelli de l'intérieur avec de très belles toiles. L'entrée était faite de deux grandes portes, une à gauche pour le Khédive et une à droite pour les dames. Il y avait trois loges fermées avec des jalousies, pour les femmes de la Cour khédiviale ${ }^{8}$. Le rideau ainsi que les fauteuils de l'orchestre avaient été commandés à Paris ${ }^{9}$. Le soir de 
l'inauguration le théâtre comptait plus de trois cents spectateurs composés de ressortissants européens, de fonctionnaires du gouvernement, de consuls, de ministres égyptiens, d'hommes d'affaires et de journalistes. Les pièces présentées étaient tirées du répertoire de Vaudeville et de celui du Palais Royal ${ }^{10}$.

Le deuxième théâtre est celui du Jardin d'al-Ezbékieh inauguré en 1873 sous la direction d'Enrico Santini. Le manque de documents concernant ce théâtre a engendré des confusions avec le théâtre de la Comédie Française. Les spectateurs ainsi que la presse les confondent en les appelant communément le théâtre du Caire ou le théâtre de la place Ezbékieh. Said Ali Ismail, présente un document des archives nationales ${ }^{11}$ permettant de distinguer les deux théâtres en donnant l'endroit précis du théâtre du jardin d'al-Ezbékieh. En effet, le document contient l'ordre khédivial pour le financement et la construction d'un nouveau théâtre à la place du Théâtre de la République et des Arts qui existait à l'époque de l'Expédition de Napoléon Bonaparte et qui avait été détruit pendant la révolte du Caire ${ }^{12}$. Le programme de ce théâtre varie entre des représentations théâtrales, et des spectacles de chant, de musique de cirque et de pantomimes.

Le lieu où l'on pouvait assister aux évènements les plus importants à cette époque-là était l'Opéra khédivial, considéré comme le monument le plus emblématique du règne d'Ismail Pacha. L'Opéra fut construit par l'architecte Avoskani, à la place du vieux palais du prince Azbak, derrière la statue d'Ibrahim Pacha. Son édification faisait partie des festivités du Canal de Suez. L'Opéra du Caire fut inauguré le $1^{\text {er }}$ novembre 1869 avec une représentation de Rigoletto de Verdi, en la présence du Khédive, de sa Cour, de la Presse, et des invités ${ }^{13}$. L'inauguration devait se faire avec la présentation de Aida de Verdi qui n’aura lieu que le 24 décembre 1887. Le khédive avait commandé cette œuvre dont le scénario, les costumes et les décors étaient esquissés par l'égyptologue Mariette pour mettre en scène le prestige de l'Égypte à travers la puissance des Pharaons. Mariette demanda au poète français, Camille du Locle de transmettre le scénario à l'un des trois grands compositeurs de l'époque et plus précisément à Gounod, Wagner ou Verdi. Le choix de du Locle se porte alors sur son ami et son collaborateur Verdi ${ }^{14}$. Celui-ci a déjà refusé de composer un hymne à l'occasion de l'ouverture du Canal, répondant qu'il n'était pas dans ses « habitudes de composer des morceaux de circonstance ${ }^{15} \gg$. Connaissant donc son caractère indépendant, Du Locle lui transmet « un scénario égyptien anonyme $\gg$, en lui demandant s'il était possible de le transformer en Opéra. Le compositeur italien a fini par accepter en imposant ses conditions financières : 150000 francs payés en or, tout en précisant qu'il ne serait pas obligé d'être au Caire pour diriger les répétitions ${ }^{16}$. 
Le khédive a dépensé des sommes colossales pour la mise en scène de la grandeur de l'Antiquité égyptienne. Mariette écrivait à son frère, en ces termes, cinq mois avant l'inauguration, parlant des moyens qu'il avait à sa disposition pour le spectacle: « Figure-toi que j’ai fait un Opéra, un grand Opéra dont Verdi fait la musique [...] Le vice-roi dépense un million. Ne ris pas. C'est sérieux ${ }^{17}$. » La première d'Aida, fut couverte par les deux journaux égyptiens Wadī al-Nìl, et al-Waqāi' al-misryya ${ }^{18}$ ainsi que par les deux critiques les plus célèbres de l'époque : M. Ernest Rever du Journal des Débats et le Docteur Filippo Filippi de la Perseveranza de Milan. Ce dernier écrit dans Musica e Musicisti en relatant ses premières impressions sur les répétitions de l'avant-dernière puis en parlant du public:

La curiosité, l'ardeur du public égyptien désireux d'assister à la première représentation d'Aida, furent telles, que depuis une quinzaine de jours, toutes les places étaient accaparées, et qu'aux derniers moments les spectateurs durent payer au poids de l'or les loges et les fauteuils [...]. Dimanche soir, le théâtre de l'Opéra était comble bien avant que l'on ne commençât: les dames occupaient en grand nombre les loges, et aucune d'elles ne vint troubler l'attention par un bavardage intempestif ou par le frou-frou de ses vêtements ${ }^{19}$.

Durant la première saison, on voit se produire sur la scène de l'opéra les célébrités de l'opéra Italien. L'Opéra khédivial continua à avoir du succès grâce aux moyens que le khédive mettait à sa disposition même après la fin des festivités du Canal. Chaque année, il y avait une saison italienne et une saison française. En 1874, pour résumer l'œuvre du khédive et son importance dans l'implantation du théâtre occidental en Égypte, Pugioli écrit les lignes suivantes :

Pour frayer un chemin au progrès et guider les peuples à se former l'esprit et le coeur, il a su associer l'utile à l'agréable; et en effet, d'après ses ordres, on a construit au Caire l'Opéra italien, des théâtres pour la comédie française ou arabe, sans se préoccuper des frais énormes résultant de l'engagement des premières célébrités artistiques, de la mise en scène de tous les chefs-d'œuvre de musique et de prose des premiers maitres et dramaturges de l'Europe. De tout temps le théâtre fut censé être un des principaux moyens d'instruction pour les peuples ${ }^{20}$.

L'histoire de l'Opéra du Caire est liée aux festivités organisées à l'occasion de l'inauguration du canal de Suez ${ }^{21}$ qui furent extravagantes. Les sommes démesurées dépensées pour cet Opéra n'étaient qu'une petite partie comparée aux autres festivités organisées pour la même occasion. Appelé depuis par les Européens «Ismail le magnifique », le khédive et ses dépenses deviennent 
même l'objet d'une pièce satirique : Le directeur du Théâtre de la Comédie Française Larose met en scène une comédie, en s'inspirant de ce fait, intitulée: C'est le Vice-roi qui paie. Elle a été présentée une seule fois, avec un grand succès. Suite au scandale qu'elle a suscité, la pièce a été arrêtée avec un reproche au directeur ${ }^{22}$. Ces dépenses attiraient également des critiques féroces de la part des paysans égyptiens qui vivaient encore dans une pauvreté extrême.

Les théâtres khédiviaux ont instauré une tradition qui sera maintenue pendant plus d'un siècle. La programmation était en majorité lyrique et exerça une grande influence sur le goût du public et sur les tendances des productions artistiques ${ }^{23}$. Outre les théâtres khédiviaux, d'autres scènes installées aux alentours offriront aux Égyptiens des représentations auxquelles ils étaient alors désaccoutumés comme les kiosques à musique, l'hippodrome, le cirque, et les salles de jeu et des spectacles de tout genre.

\section{L'initiation du public à la dramaturgie occidentale}

Avec le temps, le public et surtout les journalistes et la nouvelle génération d'intellectuels appellent à l'urgence de faire participer tous les Égyptiens aux nouvelles manifestations culturelles, aux divertissements modernes et à leur donner les outils pour déchiffrer ces formes de spectacles.

Les jardins al-Ezbékieh, jouent un rôle important dans l'initiation des Cairotes à la vie occidentale et dans la mise en place de nouvelles habitudes. Le Théâtre Khédivial de l'Opéra, qui était un modeste chapitre dans le programme des festivités du canal de Suez, devient rapidement le centre de rayonnement de la musique et du théâtre d'Europe et «accomplit un rôle principal dans l'initiation artistique des Égyptiens ${ }^{24} \gg$. Ces nouveaux divertissements changent la vie quotidienne des Égyptiens et leurs lieux de sociabilité. Désormais, les fêtes sont organisées dans des espaces privées et le théâtre se trouve dans une salle et non dans la rue ou dans les cafés. Les Égyptiens se trouvent alors témoins de l'évolution de ces lieux et des nouvelles associations entre le politique et le social dans ces mêmes lieux de divertissement. Ils étaient conviés à assister à ces représentations pour ne pas les limiter à une minorité cosmopolite composée de diplomates, techniciens et commerçants étrangers. Cependant, il n'y avait pas d'interaction entre le théâtre occidental qui se passe dans les nouvelles salles de spectacle et le théâtre égyptien traditionnel qui continue à investir les places, les rues, les cafés et les quartiers populaires bien que les annonces publiées dans les journaux ne visassent pas un public en particulier. La première annonce du journal officiel Wadì al-Nìl 
datée du 21 novembre 1870 montre bien que les nouveaux divertissements étaient destinés aux étrangers ainsi qu’aux Égyptiens :

\section{Annonce ${ }^{25}$}

Pour tout le monde et pour toute personne qui voudrait se divertir et s'amuser

Les jeux commencent dans les théâtres, c'est-à-dire les espaces de jeux occidentaux d'al-Ezbékieh, à partir de $8 \mathrm{~h}$ et quart (occidentale) du soir (c'est-à-dire environ $3 h$ du soir arabe). Voici la liste des prix pour celui qui parmi, les fils des Arabes et les respectueux dignitaires voudrait profiter de cette occasion et partager ce bonheur avant que ce soit fini. 10 francs pour chaises avant derrière l'orchestre, 70 pour les chaises arrière, 65 pour la loge d'en bas, 75 loges première classe, 52 loges deuxième classe, et 2 francs pour s'asseoir sur les escaliers de la troisième classe ${ }^{26}$.

Le programme du Cirque dirigé par le Français Théodore Rincy, ne se limitait pas à des jeux d'acrobatie. Il présentait également des spectacles de danses populaires et traditionnelles ${ }^{27}$ ainsi que des spectacles de pantomimes «étranges » pour les Égyptiens comme le montre explicitement l'annonce de l'ouverture du Cirque qui le définit entre parenthèses :

Annonce 28 $^{2}$

(Cirque français d'al-Ezbékieh sous la direction de Monsieur Rincy)

Le samedi 3 décembre occidental (premier Ramadan), le cirque présente des jeux, extraordinaires et jamais vus auparavant.

Cette année, ces jeux auront lieu pour célébrer le mois du Ramadan à partir de 8h et demi du soir.

(Deux jeux étranges jamais présentés jusqu'à nos jours.)

Le jeu dont l'intitulé signifie:

Le squelette dansant et comique

(Une sorte de pièce théâtrale interprétée par des gestes et non par la parole) ${ }^{29}$.

Le programme annoncé commence au mois du Ramadan. Le choix de cette date montre que les nouveaux divertissements européens prennent en considération les traditions et s'intègrent aux divertissements locaux de ce mois connu pour ses festivités nocturnes. L’annonce mentionne à la fin du programme qu'il y aura « des spectacles l'après-midi aussi, pour répondre à 
la demande des familles qui ne peuvent pas assister aux spectacles du soir » et exprime ainsi la volonté d'attirer toutes sortes de public.

Dans le but de permettre aux spectateurs de comprendre et de suivre le spectacle des nouveaux théâtres, Draneth Bey, propose dans une lettre adressée au khédive de traduire les livrets des opéras qui seraient représentés :

J'ai expédié il y a quelque temps à Ahmet Capitan une caisse contenant des libretti Italiens (brochures), des Opéras qui seront représentés au Caire l'Hiver prochain. Je désirerais qu'on en fit la traduction en langue Arabe, pour l'instruction des personnes qui assisteraient à leur représentation. Ces libretti ou poèmes sont en général l'auvre des poètes distingués et ce serait un service à rendre au public que de le mettre à même d'en comprendre et d'en goûter les beautés. Je vous prie donc après avoir obtenu l'autorisation de S.A de les demander à Ahmet Capitan, et vous occuper de ce travail très important à mon avis ${ }^{30}$.

Cette initiative est alors validée par le khédive et les livrets sont envoyés aux bureaux étatiques de traduction. Ainsi, trois jours avant sa représentation au Théâtre de la Comédie, La Belle Hélène d'Offenbach était traduite et imprimée en trois tomes à l'imprimerie de Būlāq $q^{31}$. Néanmoins, en dépit de cette volonté khédiviale et des efforts de traduction, le public égyptien du théâtre occidental demeure une élite, tandis que « la majorité des Égyptiens suivaient les spectacles populaires ${ }^{32} \gg$. Le journaliste français Gabriel Charmes qui a visité Le Caire, à cette époque, note que se rendre à l'Opéra n'était pas un acte spontané pour les Cairotes qui ne profitaient, en réalité que peu des spectacles européens auxquels ils assistaient. Il écrit également que les oreilles des Égyptiens trouvaient la musique européenne très bruyante et que le khédive obligeait les Pachas à louer des loges pour subventionner l'Opéra. Ils préféraient acheter alors leurs billets et laisser leurs loges vides que de s'ennuyer à voir un spectacle qu'ils ne comprenaient pas ${ }^{33}$.

La traduction des livrets ne suffisait pas pour transmettre le goût de l'Opéra aux Égyptiens. Le théâtre occidental reste donc à ses débuts un divertissement essentiellement destiné aux communautés étrangères. En revanche, c'est la presse, et particulièrement le journal $W a \bar{d}$ à al-Nìl, qui jouera un rôle principal dans la diffusion du théâtre européen dans les milieux égyptiens. Les rubriques sur les spectacles deviennent de plus en plus importantes, elles décrivent le spectacle, les acteurs, publient les livrets traduits et donnent des résumés détaillés des intrigues des pièces jouées aux théâtres de la Comédie ${ }^{34}$. Wādì al-Nìl ne se limite pas à la publication des annonces. Il explique aux lecteurs les genres et les nouveaux termes dramatiques. Par exemple pour l'article de l'inauguration, le journal a senti la nécessité d'expliquer des nouveaux 
mots comme la maison de l'Opéra et la comédie. Il mentionne même la manière avec laquelle le mot «Opéra $\gg$ se prononce $^{35}$ :

Nous avons assisté le soir du lundi à l'inauguration, avec un grand succès, d'un genre de théâtre appelé Opéra (avec une dhamma au début du mot, suivi d'un waw prolongé puis un B perse emphatique, c'est-à-dire des jeux divertissants mêlés de musique). Cette inauguration était précédée par l'ouverture d'un autre genre de théâtre ${ }^{36}$ appelé le Théâtre de la Comédie (c'est-à-dire des jeux de représentations comiques) $)^{37}$.

Le journal s'adresse également à ceux qui n'ont pas assisté aux spectacles et à ceux qui n'ont jamais été au théâtre au Caire ni dans les autres régions de l'Égypte. La deuxième saison théâtrale se caractérise par une pratique étrangère au Cairote manifeste dans la distribution des programmes et de l'affichage des annonces sur les murs de la ville. Wadì al-Nìl mentionne alors, l'étonnement des passants égyptiens à la vue de ces affiches publicitaires, publie en arabe le programme et l'annonce affichée en italien et encourage les Cairotes à venir découvrir les pièces de la nouvelle saison :

Tout le monde a vu ces jours-ci dans les rues de la ville du Caire collée sur les murs une annonce de forme étrange qui interpellait les passants, par sa beauté et son raffinement, même s'ils ne comprenaient rien de son contenu. Elle est imprimée en italien avec des caractères longs et larges, et des lettres en formes étranges et serrées. Cette affiche annonce que, par ordre de sa majesté, commencent les jeux au théâtre de l'Opéra à al-Esbékieh, dimanche prochain, premier novembre avec la représentation d'un jeu célèbre chez les communautés européennes intitulé La Favorita (c'est-à-dire la préférée). C'est une sorte de pièce théâtrale d'un genre appelé (drame) (c'est-à-dire un poème à travers lequel sont représentés des faits historiques d'une manière comique ou tragique), découpée en quatre actes, mêlée à des airs avec d'autres séquences divertissantes ${ }^{38}$, avec des danses et des danseuses européennes et d'autres arts. Nous avons publié ici le contenu de cette annonce avec l'explication des détails pour que tout le monde comprenne son sens et pour que vous soyez nombreux à y répondre ${ }^{39}$.

\section{Les aspirations des Égyptiens à un théâtre moderne arabe}

La presse joue un rôle important dans l'initiation des Égyptiens au théâtre moderne et appelle à la nécessité de la traduction du répertoire joué et son adaptation aux attentes du public égyptien. Les journalistes commencent alors à rédiger des demandes et à encourager les auteurs et toutes initiatives de traduction. Un commissaire de Police envoie une lettre à l'éditeur de Wādì al-Nìl, Abū-l-Su'ùd effenfī, pour attirer l'attention du journal sur deux 
récentes traductions de deux livrets italiens en arabe, valorisant ainsi les efforts des journalistes et des intellectuels dans la diffusion du théâtre, et en particulier Ibrahim al-Muwaylihi ${ }^{40}$ qui a publié et distribué gratuitement ces traductions :

Monsieur Ibrahim al-Muwaylīhi, est un acteur important dans l'instruction de ses compatriotes qui dépense son argent et mobilise ses idées dans la traduction des jeux théâtraux. Il les a ensuite distribués gratuitement à tous les enfants du pays qui ne connaissaient pas les langues étrangères ${ }^{41}$.

Le journal publie alors la lettre en donnant le nom du traducteur, 'Uthmān Jalāl, qui n'était pas inscrit sur les livrets ${ }^{42}$. L'article valorise ces premières traductions en les qualifiant « d'évènement littéraire important qui introduit un nouveau style d'écriture dans les journaux d'Orient et qui donnera naissance à des travaux plus laborieux ${ }^{43} \gg$. Il note également qu'elles viennent en réponse aux demandes du public désireux d'avoir les traductions des pièces qui passent aux théâtres ${ }^{44}$. Le journal nous éclaire également sur la première collaboration entre Uthmān Jalāl et Ibrahim al-Muwaylīhī, qui va être directement suivie par l'édition du journal littéraire Nuzhat al-afkār ${ }^{45}$.

$W a \bar{d}$ ì al-Nìl tente de rendre plus familiers les spectacles et de trouver des références communes aux publics arabe et occidental en faisant des connexions. Par exemple, il relève des similitudes entre Le Barbier de Séville et un conte des Mille et une Nuit: Le Barbier de Bagdād ${ }^{46}$. Pour rendre l'Opéra Moïse de Rossini plus attractif pour le public égyptien, le journal en donne un résumé et rappelle que l'histoire de Moïse est familière aussi bien pour les chrétiens ainsi que pour les musulmans, et concerne directement les Égyptiens ${ }^{47}$. Le journal révèle les noms des traducteurs comme Rifa'a at-Tahtāwī et son disciple 'Uthmān Jalāl. Un article paru en 1871, sur la traduction de La Belle Hèlène, associe pour la première fois le nom d'al-Tahtāwì à cette première traduction et à l'importation du théâtre occidental en Égypte considérée comme une œuvre civilisatrice :

Nous avons découvert un nouveau genre littéraire et un outil utile pour corriger les moeurs arabes. Cette action civilisatrice est devenue, en peu de temps, ancrée dans le goût égyptien malgré la langue étrangère des spectacles. [... ] Par ordre khédivial, le célèbre homme de lettres et l'illustre maître Rifa'at bek effendi, est celui qui a initié la diffusion de cette innovation, avec la traduction du jeu intitulé La Belle Hélène qui a brillé l'année précédente dans l'horizon des belles lettres est apparue dans les plus beaux joyaux arabes. Il a pris le soin de l'arabiser pour faire comprendre aux amateurs ${ }^{48}$ ces représentations $d u$ jeu ${ }^{49}$. 
L'article se termine par une métaphore optimiste sur les débuts du théâtre arabe moderne en comparant cette première traduction et le mouvement qu'elle a déclenché par la suite à un croissant qui va progressivement vers son accomplissement total ${ }^{50}$. Ces noms auxquels l'on doit les premières traductions, sont des figures importantes dans la réforme de l'éducation, et le fait de les assimiler aux traductions dramatiques, donne au théâtre une crédibilité, une valeur morale et un nouveau statut dans la société égyptienne. Les articles de $W a \bar{d} \bar{i}$ al-Nìl utilisent en parlant de théâtre des mots récurrents comme : « découvrir, introduction, importation, nouveau genre, innovation modernité, civilisation, morale, vertu, œuvre civilisatrice $\gg$. En associant systématiquement le théâtre à la modernisation, aux réformes khédiviales et à l'instruction du peuple égyptien, ils permettent de calmer les conservateurs qui commençaient à s'agiter à cause de la présence des femmes sur scène, et favorisent la création d'un environnement propice aux représentations et aux critiques. D'ailleurs, d'autres journaux commencent à envoyer des correspondants pour voir et commenter les pièces. Un témoignage du correspondant d'al-Jawā'ib, nous donne une idée du déroulement de ces opéras suivis par des spectateurs arabes et turcs:

Chacun d'eux tenait dans sa main un texte arabe des traductions des pièces jouées. J'ai également vu un esclave noir, avec un turban blanc, tenant à la main une traduction de Don Juan de Mozart. Ce soir-là, j'étais à la loge du directeur du théatre et il m'avait dit: Rien ne vaut le bonheur de voir les Égyptiens heureux de ces théâtres. En effet, ils ont ouvert les portes de la civilisation et le théâtre procure son côté divertissant ${ }^{51}$.

Avec la création d'un public arabe et d'une activité journalistique et critique, se forme une volonté d'avoir des représentations de pièces arabes dans les nouveaux théâtres égyptiens. Cette volonté se trouve principalement exprimée, par les traducteurs et les journalistes. Nous avons souligné l'importance de Wādī al-Nil dans la diffusion du théâtre traduit. Son éditeur luimême Abu-l-Su'ūd est le traducteur de l'Opéra Aida de Verdi. Nous relevons également la contribution de son fils Mohammed Onsy dans cette entreprise. Professeur de langue dans les écoles égyptiennes, Onsy rédige en 1872 avec Louis Farūja, enseignant de langue française à l'école des arts et métiers, une demande adressée au Khédive sous le nom de: Projet d'un théâtre national ${ }^{52}$. Cette demande officielle est appuyée par Draneth bey, le principal responsable des théâtres khédiviaux, qui avait déjà écrit au ministre Khairy Pacha pour valoriser le travail de Onsy en lui attribuant l'idée de fonder un théâtre arabe avec les mêmes privilèges que le théâtre européen en Égypte ${ }^{53}$. 
Les auteurs du Projet présentent le théâtre comme un besoin, le comparent aux autres arts, comme l'architecture, la musique, la danse, la littérature et la poésie, qui rendent la vie des humains plus agréables. Ils définissent le théâtre comme une institution utile pour l'instruction des peuples :

La création des théâtres n'est pas un fait isolé ou arbitraire. Elle est le résultat d'un besoin que personne n'ose contester [... Toutes les nations civilisées possèdent ce genre d'éducation populaire qui fait les délices de tous, grands et petits, riches et pauvres. [... Cette école charmante nous plait en nous instruisant, nous recrée en nous rendant meilleurs, et corrige, en nous amusant, l'âpreté de nos mours. [...] La création des théâtres est donc une cuvre éminemment utile parce qu'elle est et doit être morale ${ }^{54}$.

Après les remerciements, les éloges faits au khédive Ismail « Magnanime fondateur des beaux-arts en Égypte », et l'énumération de toutes ses réformes, les auteurs du Projet demandent son encouragement et son accord. Ils formulent explicitement leur objectif: « Le but que nous proposons est la création définitive d'un Théâtre Arabe qui prendra officiellement le nom de Théâtre National ». Ils proposent un plan détaillé composé de deux grandes parties : les éléments intrinsèques et extrinsèques. Les éléments intrinsèques intègrent la Direction Générale (le patronage du Khédive), la Direction Particulière (Draneth Bey), les Artistes, L'Orchestre, Les Employés subalternes, et Le Répertoire. Les éléments extrinsèques intègrent le local, les costumes, le temps (une à deux pièces par semaine), la recette, et les frais. Il s'agit d'un théâtre-école qui présenterait « le double avantage de dresser les sujets, préparer les artistes et donner en même temps des représentations. » La troupe sera composée de vingt jeunes acteurs-apprentis ( 6 filles et 14 jeunes gens). Ils doivent maîtriser la langue arabe et connaître une langue étrangère, de préférence la langue française. Ils doivent remplir certaines conditions pour être admis et ils doivent se soumettre au règlement du théâtre. Ils proposent un théâtre principalement lyrique. Ce théâtre école aurait donc au programme des cours de déclamation, de musique vocale, des cours de littérature arabe ce qui implique d'embaucher des professeurs et des traducteurs pour former le répertoire.

La traduction, telle qu'elle est présentée dans le projet, est étroitement liée à la censure. En effet, le répertoire se compose de traductions qui doivent être révisées et admises par « une commission de littérature et des gens de l'art ${ }^{55} \gg$. La programmation du théâtre impose également la présentation des traductions puis des pièces originales. Pour appuyer leur initiative, les auteurs du Projet suggèrent que le théâtre arabe soit attaché à la Direction générale 
des théâtres khédiviaux donc soumis à une grande censure. Cette alliance de pouvoir et de censure rendrait « le contrôle plus facile » et permettrait d'éloigner de la scène quelques expériences locales libres :

Le répertoire est une chose essentielle de tout bagage théâtral. Il n'en peut être ainsi dans une chose qu'il s'agit de créer. C'est le passé qui prépare les ressources de l'avenir et, ici, le passé ne compte pas. Malgré ce vide et grâce à un programme intelligent, le répertoire ne tardera pas à se faire. S'il plait à son Altesse de donner l'exequatur à notre projet, nous nous mettrons à l'ouvre. Dans le principe, le répertoire sera composé de Pièces traduites, puis des pièces du cru. Loin de nous la pensée de débuter par des pièces faites sur place et n'ayant pas subi aucun contrôle, aucune censure. Nous débuterons par des pièces traduites soit du français, soit de l'italien et puis viendront les pièces locales.

Le projet de Mohammed Onsy et de Louis Farūgia, conçoit le théâtre national arabe comme un théâtre officiel soumis au contrôle du gouvernement et non comme un théâtre critique envers la cour khédiviale et la société égyptienne. Leur initiative appuyée par Draneth serait peut-être une réponse aux premières pièces locales inspirées conjointement par le répertoire occidental et le théâtre populaire qui échappe justement, en partie, au contrôle du gouvernement. D'ailleurs, le soutien de Draneth à ce théâtre officiel est signalé par James Sanua dans Les Tribulations de Molière d'Égypte, comme une tentative pour l'éloigner de la scène égyptienne ${ }^{56}$. En effet, ce Projet compte donner naissance à un répertoire du théâtre arabe à partir de traductions révisées de pièces occidentales italiennes et françaises. Ses auteurs qualifient de « vide » l'héritage théâtral arabe. Néanmoins, cette initiative a le mérite de formuler, officiellement et pour la première fois, la demande de « théâtre national » et de s'inscrire dans les démarches qui ont permis l'émergence d'un théâtre moderne de langue arabe ${ }^{57}$.

Ainsi, on voit que parallèlement aux expériences dramatiques des pionniers du théâtre arabe moderne notamment Ya'qūb Sanū' et 'Uthmān Jalāl, il existait des démarches « annexes » permettant d'avoir une vue d'ensemble sur les différentes initiatives politiques, culturelles, et sociales qui ont permis à la société égyptienne d'exprimer son besoin de créer son propre théâtre et d'y injecter une expression qui lui est spécifique et particulière. En effet, les traductions des œuvres et des annonces, la recherche et la construction d'un langage dramatique arabe, ainsi que l'intégration des sorties aux théâtres dans les mondanités et dans les pratiques culturelles et politiques ont permis- 
la mise en place d'une tradition théâtrale directement inspirée de la culture occidentale.

\section{Bibliographie}

Arnaud Jean-Luc, Le Caire: mise en place d'une ville moderne 1867-1907, Paris, Actes Sud, 1998.

Attia Abul Naga, Les Sources françaises du théâtre égyptien (1870-1939), Alger, SNED, 1972.

Douin G., Histoire du règne du khédive Ismail, tome III, L'Empire africain, Le Caire, Institut français d'archéologie orientale, 1936.

Garfi Mohammed, Musique et spectacle, Le théâtre lyrique arabe, esquisse d'un itinéraire (1847-1975), Paris, L'Harmattan, 2009.

Hallaq Boutros et Toelle Heidi (dir.), Histoire de la littérature arabe moderne, tome I, 1800-1945, Paris, Actes Sud, 2007.

Hanotaux Gabriel, Histoire de la nation égyptienne, tome VI, L'Égypte de 1801 à 1882, Mohammed Ali et sa dynastie jusqu'à l'occupation anglaise, par F. CharalesRoux, Paris, Libraire Plon, 1936.

Ismail Said Ali, Histoire du théâtre en Égypte au XIX siècle, Le Caire, Al-hay-a al-miçryya al-āmma lil kitāb.

Louca Anouar, Voyageurs et écrivains égyptiens en France au XIX siècle, Paris, Éd. Didier, 1970.

Prince Osman Ibrahim, Kurhan Caroline et Ali, Mébémet Ali le grand, Mémoires intimes d'une dynastie (1805-2005), Paris, Maisonneuve et Larose, 2005.

Pugioli Joseph, Précis expositif du Gouvernement de son altesse Ismail Ier khédive d'Égypte, (Très respectueusement dédié à S.A. Mohamed Terwfik Pacha Prince héréditaire), 13 juin 1874, Éd. Alexandrie d'Égypte 1874.

Sadgrove P.C, The Egyptian Theatre in the Nineteenth Century 1799-1882, Lebanon, Ithaca Press, 1996.

—_, Al-Masrah al-miçrī fi al-Qarn al-tāsi’'ašar, (en arabe), Traduit par Amine al-'Ayoutī, présenté et annoté par Ismail Said Ali, Le Caire, Publications centre national du théâtre de la musique et des arts populaires, 2007.

Sammarco Angelo, Histoire de l'Égypte moderne, tome III, Le règne du khédive Ismail de 1863 à 1875, Le Caire, Institut français d'archéologie orientale, 1937.

Sidky Abdel Rahman, Opéra khédivial du Caire: L'Égypte et Verdi, Le Caire, 1951. Sole Robert, L'Égypte, passion française, Paris, Éditions du Seuil, 1997.

L'héritage du théâtre, Publication du centre national du théâtre, de la musique et des arts populaires, $n^{\circ} 1$, juin 2000 , Le Caire. 


\section{Annexes}

$\mathrm{N}^{\circ} 1$ : Wādī al-Nìl, 21/11/1870, no 20

\section{اعلان}

لجميع الناس و كل من أراد التروح و الإيتناس

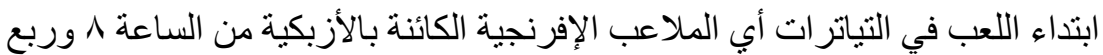

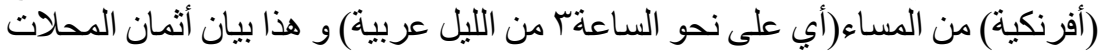

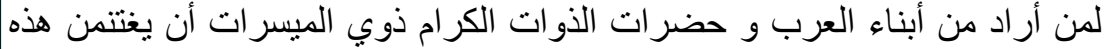

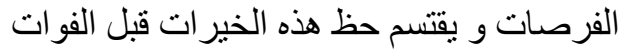

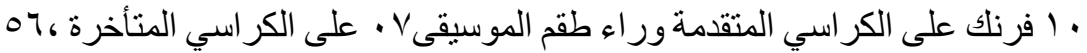

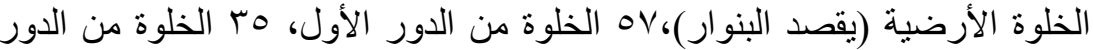

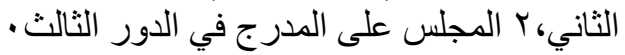

$\mathrm{N}^{\circ} 2$ : Wādī al-Nil 5/11/1869, ${ }^{\circ} 25$

ليلة الاثنين الماضي (المو افق / / / / 1 (97) حصل اول الافتتاح مع غاية النجاح

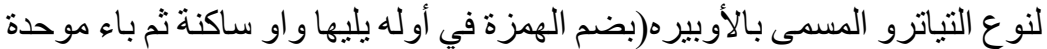

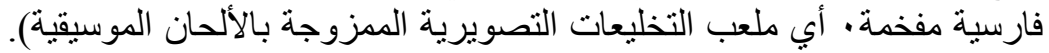

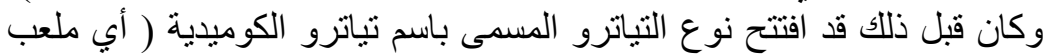
التخليعاتالمضحكة). 
No 3 : Wādì al-Nìl, 3 décembre. 1869 I'lān

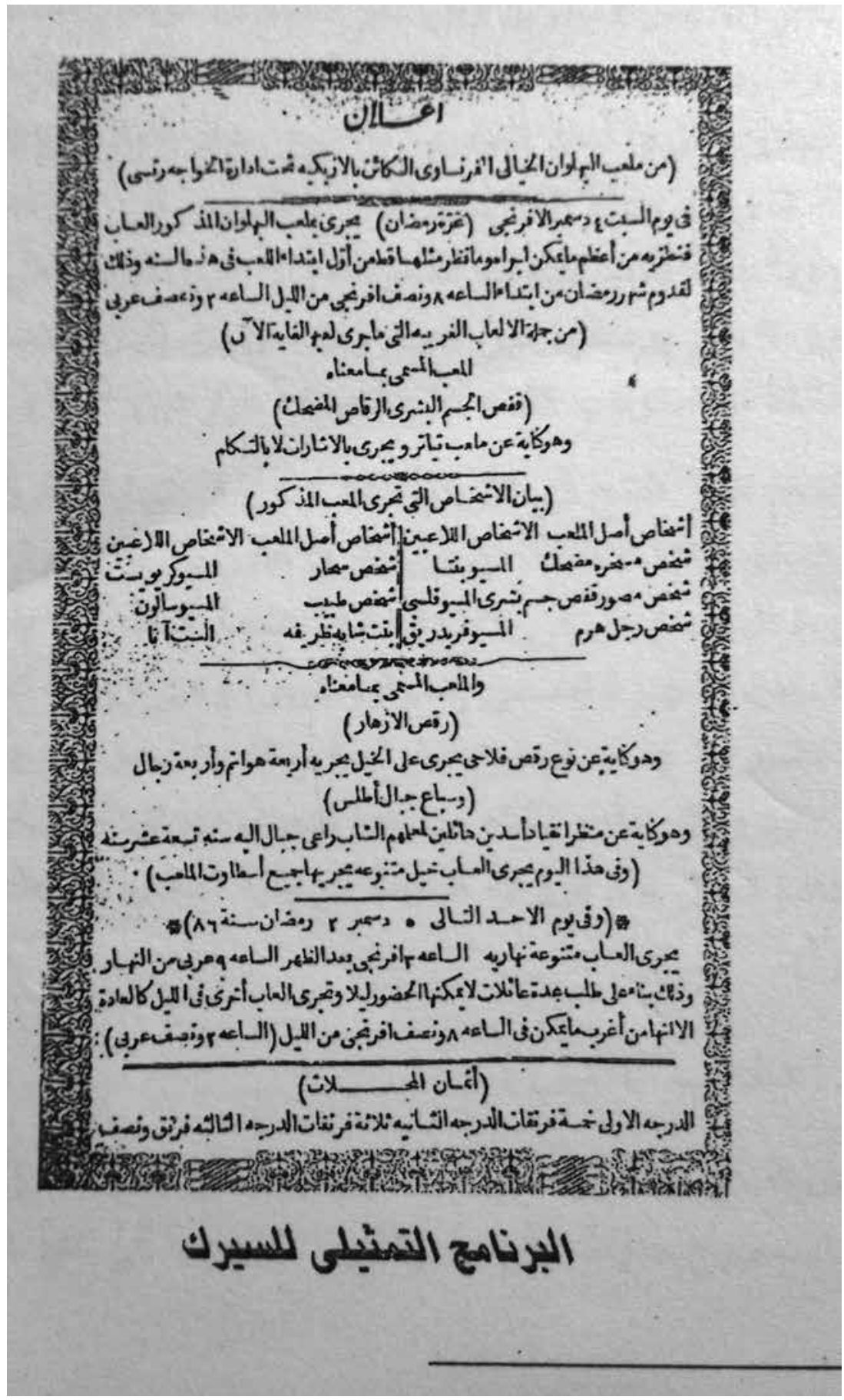


$\mathrm{N}^{\circ} 4$ : Wādī al-Nìl, 5 novembre, 1869, $\mathrm{n}^{\circ} 28$

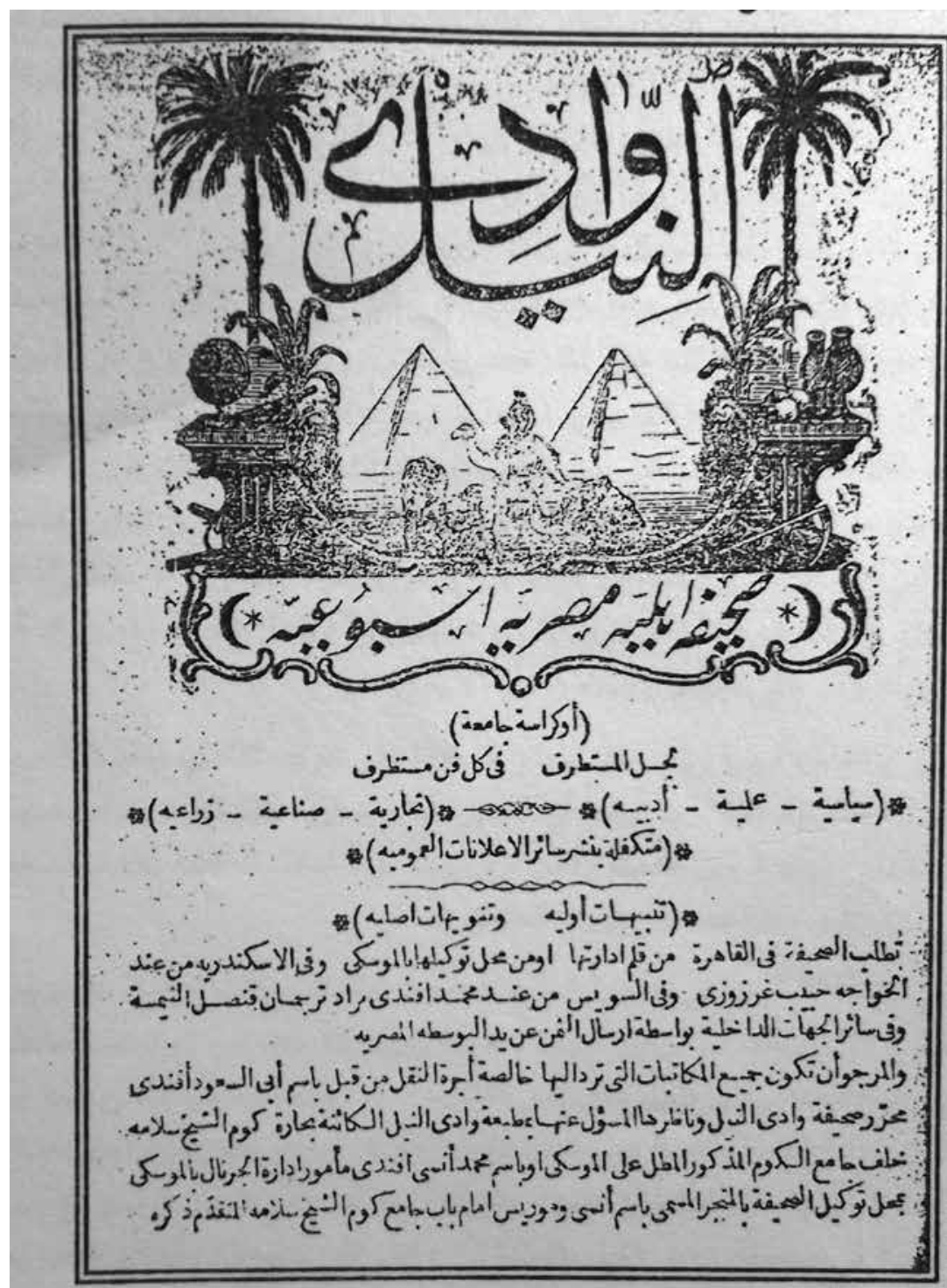

مورة فلاف مجالة (والد الثنبل

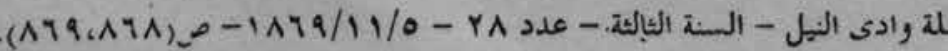




\section{Notes}

1. Nous tenons les informations sur la vie et le règne du Khédive Ismail des études suivantes:

Angelo Sammarco, Histoire de l'Égypte moderne, tome III, Le règne du khédive Ismail de 1863 à 1875, Le Caire, L'Institut français d'archéologie orientale, 1937.

G. Douin, Histoire du règne du khédive Ismail, tome III, L'Empire africain, Le Caire, L'Institut français d'archéologie orientale, 1936.

Gabriel Hanotaux, Histoire de la nation égyptienne, tome VI, L'Égypte de 1801 à 1882, Mohammed Ali et sa dynastie jusqu'à l'occupation anglaise, par F. Charales-Roux, Paris, Libraire Plon, 1936.

Anouar Louca, Voyageurs et écrivains égyptiens en France au XIX siècle, Paris, Éd. Didier, 1970.

Prince Osman Ibrahim, Caroline et Ali Kurhan, Méhémet Ali le grand, Mémoires intimes d'une dynastie (1805-2005), Paris, Maisonneuve et Larose, 2005.

2. Robert Sole, L'Égypte, passion française, Paris, Éditions du Seuil, 1997, p. 217.

3. Jean-Luc Arnaud, Le Caire: mise en place d'une ville moderne 1867-1907, Paris, Actes Sud, 1998, p. 50-67.

4. Abul Naga Attia, Les Sources françaises du théâtre égyptien (1870-1939), Alger, SNED, 1972, p. 64-65.

5. Voir annexe $n^{\circ} 4$.

6. Cette place doit son nom au Palais de Azbak al-zāhirī construit en 1476.

7. L'héritage du théâtre, Publication du centre national du théâtre, de la musique et des arts populaires, $n^{\circ} 1$, juin 2000, Le Caire, p. 83-88.

8. Said Ali Ismail, Histoire du théâtre en Égypte au XIX siècle, Le Caire, Al-hay-a al-miçryya al-āmma lil kitāb, 2005, p. 37.

9. Robert Sole, op. cit., p. 220.

10. P.-C. Sadgrove, The Egyptian Theatre in the Nineteenth Century 1799-1882, Lebanon, Ithaca Press, 1996, p. 46.

11. Archives nationales: document 1/1/39, p. 95. In Histoire du théâtre en Égypte au XIX siècle, op. cit., p. 42.

12. Said Ali Ismail, op. cit., p. 40-50.

13. Said Ali Ismail, op. cit., p. 100.

14. Abdel Rahman Sidky, Opéra khédivial du Caire: L'Égypte et Verdi, Le Caire, 1951.

15. Lettre conservée dans les archives du Théâtre royal de l'Opéra du Caire, sub. No. I, In, L'Égypte et Verdi.

16. Ibid.

17. Édourd Mariette, Mariette pacha. Lettres et souvenirs personnels, Paris, 1904. Cité par Robert Sole, op. cit., p.239.

18. P.C. Sadgrove, op. cit., p. 112.

19. Traduction dans Verdi de Arthur Pougin, p. 225-227, In L'Égypte et Verdi, op. cit.

20. Joseph Pugioli, Précis expositif du Gouvernement de son altesse Ismail Ir khédive d'Égypte, (très respectueusement dédié à S.A. Mohamed Tewfik Pacha Prince héréditaire), 13 juin 1874, Éd. Alexandrie d'Égypte 1874. 
21. Pour les dépenses, le programme des fêtes et les invités européens, les hôtes royaux et princier, Sammarco donne plusieurs références dont: A. Mariette, Itinéraire des invités aux fêtes de l'inauguration du Canal de Suez, Le Caire-Alexandrie 1869. De Lesseps, Lettres, journal et documents, t. V, Ch. Taglioni, Deux mois en Égypte, Journal d'un invité du Khédive, Paris, 1870. Également le témoignage de Charles Blanc, Voyage de la Haute-Égypte, Paris 1876 : « Nous n'avions aucune dépense à faire, aucune. Les plus beaux hôtels avaient ordre de nous recevoir et de nous traiter comme les hôtes du khédive. Avec une générosité incomparable, le Vice-Roi avait mis à notre disposition des voitures, des chevaux, des ânes, des âniers. Qu'un prince ait été assez magnifique et assez riche pour inviter cent personnes à venir de la France, de l'Espagne, de l'Allemagne, de la Suède, faire à ses frais l'expédition de la Haute-Égypte et pour inviter ensuite neuf cent personnes à l'inauguration de l'Isthme de Suez, en leur offrant, avec la gratuité d'un si long trajet, l'honneur d'être hébergés, logés, amusés, guidés et instruits à ses dépens, c'est là un trait d'hospitalité qui, certainement n'a pas eu d'exemple, et qui probablement n'aura point d'imitateurs ». In Sammarco, op. cit., p. 194.

22. Chaillé-Long (1912), 35, Cité par Sadgrove, op. cit., p. 47.

23. Mohammed Garfi, Musique et spectacle, Le théâtre lyrique arabe, esquisse d'un itinéraire (1847-1975), Paris, l'Harmattan, 2009, p. 78-82.

24. Ibid., p. 78.

25. Voir annexe $n^{\circ} 1$.

26. Wādī al-Nī1, 21/11/1870, n 20, p. 8. Cité par A.S. Ismail, Histoire du Théâtre en Égypte au XIX siècle, op. cit., p. 26.

27. Said Ali Ismail, Histoire du Théâtre en Égypte au XIXe siècle, op. cit., p. 32.

28. Voir annexe $\mathrm{n}^{\circ} 2$.

29. I'lān (annonce), Wãdì al-Nì̀l, Le Caire, 3 décembre 1869, dernière page, In Said Ali Ismail, Histoire du Théâtre en Égypte au XIX siècle, op.cit. p.33. Également cité et traduit par GARFI, op. cit., p. 80.

30. Lettre datée: Paris, 27 juillet 1869, de Draneth à H.E Khairy Bey, In Dār al-wathā'iq, Ahd Isma'il. 127, document, 80/5. Cité par Sadgrove, op. cit., p. 48.

31. Sadgrove op. cit., p. 47.

32. Boutros Hallaq et Heidi Toelle (dir.), Histoire de la littérature arabe moderne, tome I, 1800-1945, Paris, Actes Sud, 2007. Monica Ruocco «La Nahda par l'Iqtibās (1): Naissance du théâtre arabe », pp.151-186.

33. G. Charrmes, (1883), Five Months at Cairo and in Lower Egypt, London, Richard Bentley and Son. In P.C. Sadgrove, op. cit., p. 54-55.

34. D'après l'article du 29/11/1869, cité par Said Ali Ismail, Histoire du Théâtre en Égypte au XIX $X^{e}$ siècle, op. cit., p. 117.

35. Voir annexe $\mathrm{n}^{\circ} 3$.

36. Le texte arabe emploie le mot « tiatro " pour théâtre et «at-tyātrāt " pour les théâtres.

37. Wādì al-Nil 5/11/1869, n²5, In Said Ali Ismail, Histoire du Théâtre en Égypte au XIX siècle, op. cit., p. 70.

38. Takbli'at-mussalya, voir le texte arabe en annexe.

39. Wādì al- Nil, 25/10/1870, n 54, In Said Ali Ismail, Histoire du théâtre en Égypte au XIX siècle, op. cit., p. 78. 
40. Ibrahim al-Muwaylīhī, critique politique, journaliste et auteur de Hadìth 'Issā IbnūHichàm (Ce que nous conta 'Issā Ibn Hichām).

41. Wādī al-Nil, 14/11/1870, note no 2, p. 120. In P. Sadgrove, Al-Masrah al-miçrī fi al-Qarn al-tāsi' ašar (en arabe), traduit par Amine al-' Ayoutī, présenté et annoté par Said Ali Ismail, Le Caire, Publications centre national du théâtre de la musique et des arts populaires, 2007.

42. Said Ali Ismail, In Le théâtre égyptien au XIXe siècle, op.cit. note $\mathrm{n}^{\circ} 1, \mathrm{p} .121$.

43. Ibidem. Note $\mathrm{n}^{\circ}$ 3, p. 123.

44. Sadgrove, op. cit., p. 60.

45. Selon la description de l'article, la dernière page du premier livret, marque qu'il s'agit de la traduction de La Favorite de Gaetano Donizetti, présenté le 1er novembre 1870 pour l'ouverture de la nouvelle saison de l'Opéra Khédivial. Le deuxième livret intitulé "La deuxième nuit de l'Opéra" est une traduction du Il Barbiere di Siviglia de Rossini présenté avec le livret de C. Stesbini au Caire le 3 novembre 1870. En publiant les livrets avec le nom de leur traducteur, $W a \bar{d} d \bar{l}$ al- $N \bar{\imath} l$, nous renseigne sur les premières traductions d'Uthmān Jalāl.

46. Sadgrove, op. cit., p. 62.

47. Wādì al-Nil,24/2/1871, no 84, Said Ali Ismail, Histoire du théâtre égyptien, op.cit. p.78.

48. Ces représentations du jeu, ou représentations/images jouées pour dire pièces de théâtre ou opéra ou spectacle.

49. Ismail Said Ali. In Le théâtre égyptien au XIXe siècle, op. cit., note $\mathrm{n}^{\circ} 1, \mathrm{p} .174$.

50. Wãdì al-Nil, 6/1/1871.

51. Al-Jaw'ib, 12 avril 1872, cité par Sadgrove, op. cit., p. 55.

52. Le Caire, 15 mars 1872. Sadgrove, op. cit., Appendix no 3, p. 186-195.

53. Sadgrove, op. cit., p. 105.

54. Le texte original publié par Sadgrove est écrit en français. (Ismail Said Ali a publié une traduction et non pas le texte original comme il avait traité tous les autres documents présentés dans son ouvrage cité plus haut.)

55. Projet d'un théâtre national, op. cit., p. 192.

56. Voir l'avis de Sadgrove, op. cit., p. 175: Draneth appuit la demande de Onsy, pour éloigner Sanua et son théâtre: « c'est Draneth qui a suggéré l'idée à Onsy, pour pouvoir ainsi diriger le théâtre arabe ».

57. Le projet reste sans réponse du khédive, ou plutôt une réponse négative, puisqu'il n'y a pas eu de théâtre construit spécialement pour le théâtre moderne de langue arabe. Le premier institut étatique d'art dramatique dans le monde arabe était en Égypte 1930, In Le théâtre égyptien au XIX $X^{\mathrm{e}}$ siècle, op. cit, note $\mathrm{n}^{\circ} 1, \mathrm{p} .174$. 